$11-1-1990$

\title{
Using Refraction Caustics to Monitor Evaporation of Liquid Drop Lenses
}

James A. Lock

Cleveland State University, j.lock@csuohio.edu

Jearl D. Walker

Cleveland State University

James H. Andrews

Follow this and additional works at: https://engagedscholarship.csuohio.edu/sciphysics_facpub

Part of the Physics Commons

How does access to this work benefit you? Let us know!

\section{Publisher's Statement}

This paper was published in Applied Optics and is made available as an electronic reprint with the permission of OSA. The paper can be found at the following URL on the OSA website: http://www.opticsinfobase.org/ao/abstract.cfm?URI=ao-29-31-4599. Systematic or multiple reproduction or distribution to multiple locations via electronic or other means is prohibited and is subject to penalties under law.

\section{Original Citation}

Lock, James A., Jearl D. Walker, and James H. Andrews. "Using Refraction Caustics to Monitor Evaporation of Liquid Drop Lenses." Applied Optics 29 (1990): 4599-4607.

\section{Repository Citation}

Lock, James A.; Walker, Jearl D.; and Andrews, James H., "Using Refraction Caustics to Monitor Evaporation of Liquid Drop Lenses" (1990). Physics Faculty Publications. 92.

https://engagedscholarship.csuohio.edu/sciphysics_facpub/92

This Article is brought to you for free and open access by the Physics Department at EngagedScholarship@CSU. It has been accepted for inclusion in Physics Faculty Publications by an authorized administrator of EngagedScholarship@CSU. For more information, please contact library.es@csuohio.edu. 


\title{
Using refraction caustics to monitor evaporation of liquid drop lenses
}

\author{
James A. Lock, Jearl D. Walker, and James H. Andrews
}

\begin{abstract}
Irregularities in the perimeter of a water droplet adhering to a vertical pane of glass cause perturbations in the curvature of the droplet surface. When laser light passes through such a droplet, the perturbations produce a far field refraction caustic, which is a section of the caustic known as the parabolic umbilic in the catastrophe theory classification. As the water evaporates and the droplet surface curvature changes, the section of the parabolic umbilic caustic on the viewing screen also changes. We determine the evolution of curvature of the droplet surface by observing the evolution of the far field caustic and the locations on the droplet responsible for the various features of the caustic.
\end{abstract}

\section{Introduction}

Optical caustics have been defined in three equivalent ways. When light is deflected (i.e., reflected or refracted) from an interface, an optical caustic is the locus of the points at which the intensity of the deflected rays becomes infinite. ${ }^{1}$ It is also the envelope of the deflected rays ${ }^{2}$ and the locus of the principal centers of curvature of the deflected wavefronts. ${ }^{3}$ Since the shape of a caustic is related to the shape of the deflecting surface which causes it, various properties of an irregularly shaped transparent object may be determined by the analysis of the far field refraction caustic that it produces.. The purpose of this paper is to determine the changes in the curvature of the surface of a water droplet adhering to a vertical pane of glass as it evaporates by analyzing its far field caustic.

There are two approaches to analysis of optical caustics. The first approach employs the eikonal approximation for the deflected wavefield and expresses the caustic in terms of the metric properties of the deflecting surface..$^{3-7}$ This approach has been widely used in the analysis of the aberrations of imaging systems. The second approach classifies and describes certain types of caustics using the mathematics of catastrophe theory. 8,9 This second approach is the paraxial approximation to the first approach and directly gives

When this work was done all authors were with Cleveland State University, Physics Department, Cleveland, Ohio 44115; J. H. Andrews is now with Case Western Reserve University, Cleveland, Ohio 44106.

Received 14 July 1989.

0003-6935/90/314599-09\$02.00/0.

(C) 1990 Optical Society of America. both the caustic and the diffraction structure which surrounds it. Although the second approach gives simple descriptions to only a subset of the caustics analyzable using the first approach, the subset is sufficiently large and the simplifications are sufficiently great that the second approach has enjoyed substantial success in describing many diverse natural phenomena. ${ }^{10-15}$

Because the observed caustic in our experiment appears to be a progression between different sections of the caustic known as the parabolic umbilic in the catastrophe classification scheme, the observations are most easily and naturally described by the second approach, as we employ here. The relevant techniques from catastrophe theory are reviewed briefly in Sec. II. Our visual observations of the caustic are described in Sec. III. The evolution of the caustic during the evaporation of the droplet is analyzed in Sec. IV. Lastly, the curvature of the droplet surface is determined from the evolution in Sec. V.

\section{Optical Caustics and Catastrophe Theory}

We consider plane waves of wavelength $\lambda$ propagating along the $z$-axis toward the $z=0$ entrance plane where there is a transparent refracting material with refractive index $n$ and thickness $t(x, y)$. The light refracted through the material is observed in the far field at the point $\left(x_{0}, y_{0}\right)$ on a viewing screen a distance $z_{0}$ from the entrance plane. The advance in the phase of a wave propagating from $(x, y)$ in the entrance plane to $\left(x_{0}, y_{0}, z_{0}\right)$ on the viewing screen is

$$
\phi\left(x, y, x_{0}, y_{0}, z_{0}\right)=\frac{2 \pi}{\lambda}\left[(n-1) t(x, y)+\frac{x^{2}}{2 z_{0}}+\frac{y^{2}}{2 z_{0}}-\frac{x x_{0}}{z_{0}}-\frac{y y_{0}}{z_{0}}\right]
$$

in the paraxial approximation. ${ }^{14,16}$ The optical caus- 
tic that appears on the viewing screen may be determined by the following procedure. The electric field on the viewing screen is written as a diffraction integral over the entrance plane coordinates as

$$
E\left(x_{0}, y_{0}, z_{0}\right)=\int d x d y \exp \left[i \phi\left(x, y, x_{0}, y_{0}, z_{0}\right)\right] .
$$

The source of the caustic is the curve in the entrance plane given by the vanishing of the Hessian of $\phi$, i.e.,

$$
H(\phi) \equiv\left(\frac{\partial^{2} \phi}{\partial x^{2}}\right)\left(\frac{\partial^{2} \phi}{\partial y^{2}}\right)-\left(\frac{\partial^{2} \phi}{\partial x \partial y}\right)^{2}=0 .
$$

This curve in the entrance plane is then mapped into the caustic observed on the viewing screen by the transformation

$$
\frac{\partial \phi}{\partial x}=0, \quad \frac{\partial \phi}{\partial y}=0
$$

This prescription is known as the "optical path matrix"17 or "singularity of the gradient map"18 prescription and has been shown ${ }^{9,17,18}$ to be equivalent to the standard definitions of the caustic given in Refs. 1-3.

If $\phi$ is a polynomial of low degree in $x$ and $y$, the shapes of the resulting caustics and the wave interference structure that surrounds them may be easily calculated. In catastrophe theory, such caustics are named and studied. The names and photographs of several of them are given in Refs. 8, 9, and 19.

In an analysis of an evaporating water droplet, one useful property of a caustic is the connection between its shape and the shape of its $H(\phi)=0$ source curve in the entrance plane. ${ }^{20}$ If the source curve locally resembles a line, a parabola, an ellipse, a hyperbola, an $x$ shape, or a cubic, the curve maps via Eq. (4) into caustics that are known as the fold, cusp, elliptic umbilic, hyperbolic umbilic, the singular section of a hyperbolic umbilic, and a swallowtail, ${ }^{21}$ respectively.

In an analysis of experiments similar to the one performed here, Nye found that the solution to the differential equation describing the equilibrium shape of water droplet spanning a vertical template hole is identical to the shape of the refracting surface that produces a portion of the caustic known as the parabolic umbilic in the catastrophe theory classification. ${ }^{11}$ Our experiment differs from that of Nye in that he explored different sections of the parabolic umbilic by varying the position of the viewing screen in the near field while maintaining the shape of the droplet. In our case, we allow evaporation to change the drop's shape and keep the position of the viewing screen fixed in the far field.

The phase function of Eqs. (1)-(4) for the parabolic umbilic is

$$
\phi(x, y)=\frac{\epsilon}{4} y^{4}+\eta x^{2} y+\gamma x^{2}+\delta y^{2}-\alpha x-\beta y .
$$

The equation of the curve in the $x-y$ plane of the water droplet that is mapped into the parabolic umbilic on the viewing screen is given from Eq. (3) by

$$
(\eta y+\gamma)\left(3 \epsilon y^{2}+2 \delta\right)=2 \eta^{2} x^{2} \text {. }
$$

Using Eq. (4), the 2-D section in $\alpha-\beta$ space of the parabolic umbilic is given parametrically by

$$
\begin{aligned}
& \alpha=2 x(\eta y+\gamma), \\
& \beta=\eta x^{2}+y\left(\epsilon y^{2}+2 \delta\right),
\end{aligned}
$$

where $x$ and $y$ are solutions to Eq. (6).

When the coordinates $x$ and $y$ are scaled in such a way that the magnitudes of $\epsilon$ and $\eta$ are unity, the shape of the caustic depends on the parameters $\alpha, \beta, \gamma$, and $\delta$. In that case, the parabolic umbilic's structure lies in the 4-D space of these parameters. Since $\alpha$ and $\beta$ are proportional to the viewing screen coordinates $x_{0}$ and $y_{0}$, the caustic's cross section in an $\alpha-\beta$ plane is the same as on the screen. That fact allows the crosssectional shapes to be organized. For given values of $\gamma$ and $\delta$, the shape in $\alpha-\beta$ space is determined and associated with the values. The procedure is repeated for other points in the $\gamma-\delta$ plane. Such an organization, with the constraint that $\epsilon=\eta=1$ is shown in Fig. 7 of Ref. 11 and in Fig. 5.24 of Ref. 22.

\section{Observation of the Refraction Caustic of the Evaporating Water Droplet}

Some of the experimental observations described here have already been reported elsewhere by one of us. $^{23}$ A portion of a commercially manufactured plastic template was glued to a clean microscope slide. The template was $0.76 \mathrm{~mm}$ thick, and the portion used had circular holes $2.5,3.0$, and $3.5 \mathrm{~mm}$ in diameter. Droplets of water were placed into a template hole with a syringe until the water in the hole spanned it and behaved like a small converging lens. The microscope slide was then placed vertically in the expanded beam of a $60-\mathrm{mW} \mathrm{He}-\mathrm{Ne}$ laser with the curved side of the droplet facing away from the laser. A caustic consisting of a number of cusps joined by folds appeared on a viewing screen in the far field $2 \mathrm{~m}$ away.

Each cusp sprung from an irregularity in the droplet's perimeter that resulted from either minute scratches in the template, grease or dust motes at the junction of the water and the edge of the hole, or imperfections in the shape of the hole. Similar caustic patterns produced by raindrops on a window pane have been described by Minnaert ${ }^{24}$ and have been analyzed for both horizontal ${ }^{10}$ and vertical ${ }^{11}$ glass substrates by Nye.

The vertical microscope slide was rotated in its own plane until the prominent cusp was located at the bottom of the caustic pattern on the viewing screen. As the water slowly evaporated, the caustic at the bottom of the viewing screen came to dominate the entire field of view and its shape evolved as shown in Figs. 1(a)-(f).

The evolution was studied in four ways. First, the caustic's shape was recorded and its major features were identified with certain elementary caustics in the catastrophe theory classification. Second, the positions on the viewing screen of the major features were measured during the evolution. Third, the locations on the water droplet responsible for those features were determined. Fourth, the focal length of the water droplet lens was measured at various times during the evolution. 


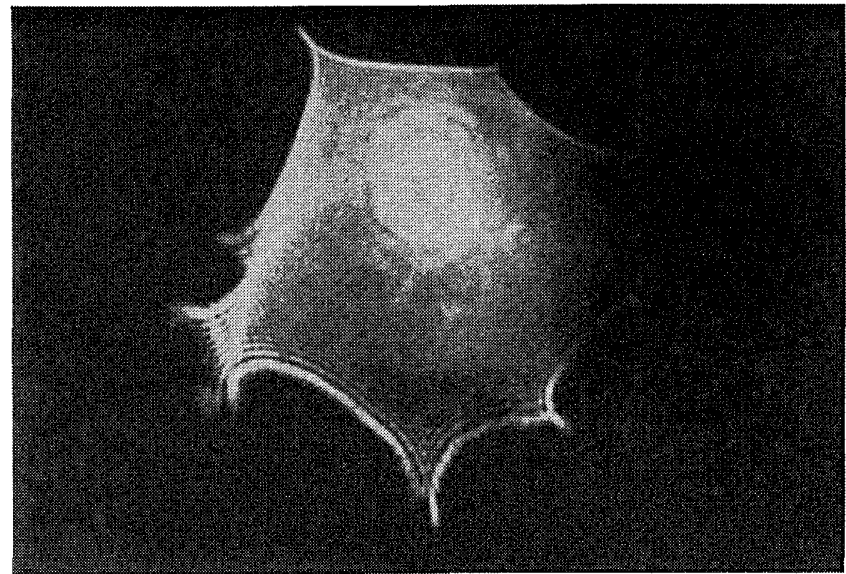

(a)

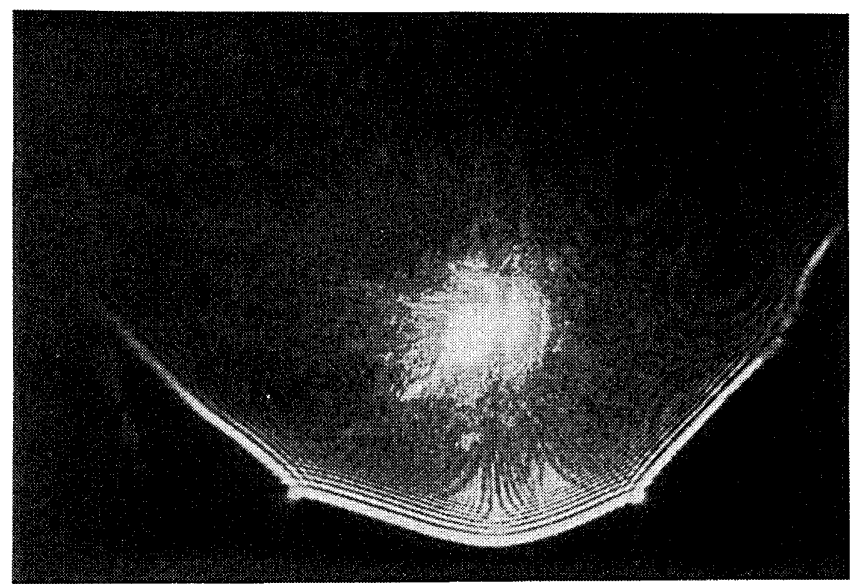

(c)

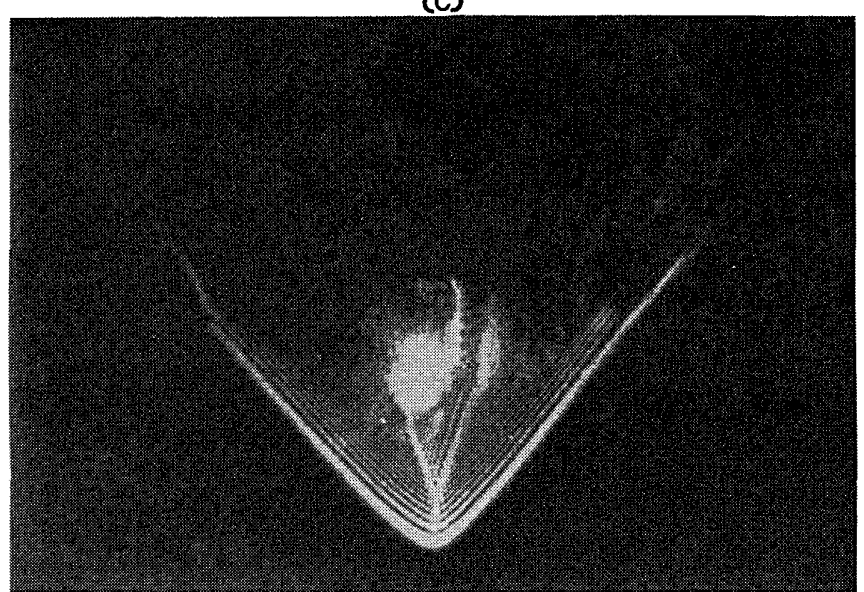

(e)

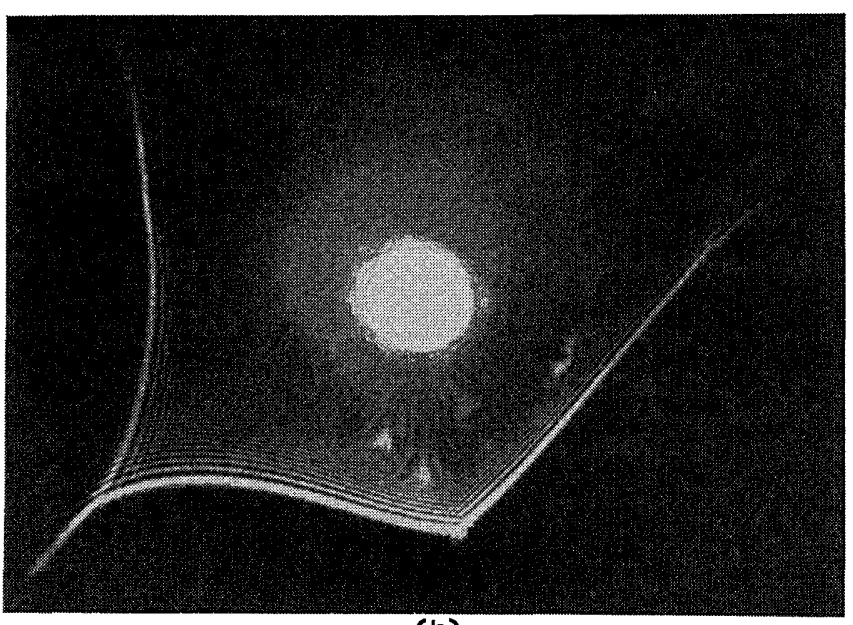

(b)

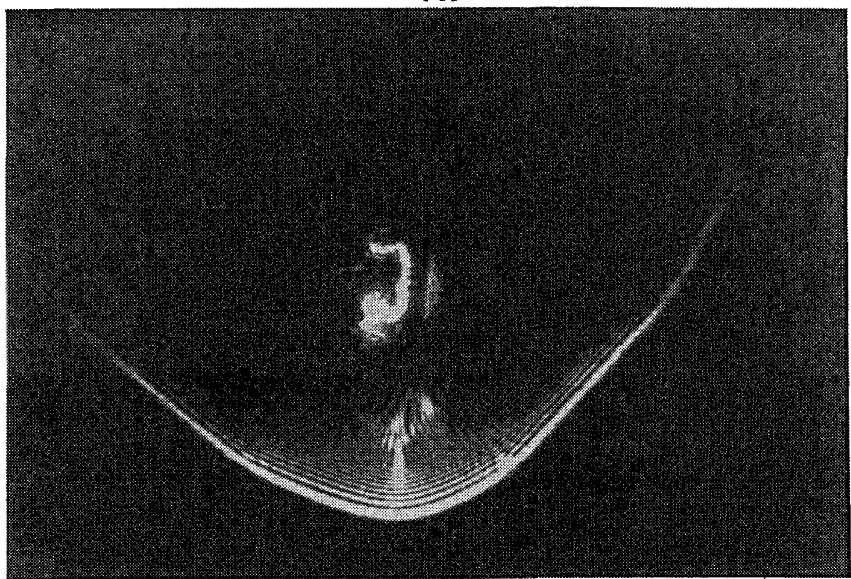

(d)

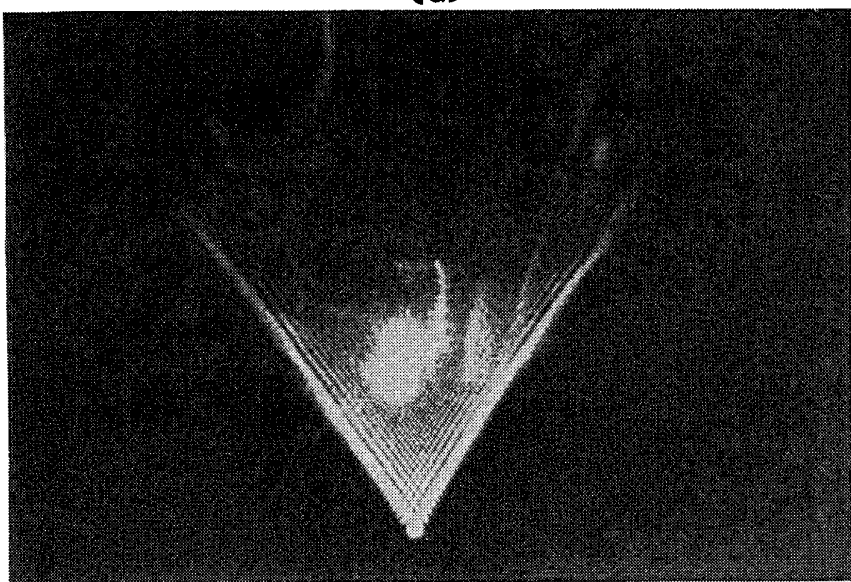

(f)

Fig. 1. Evolution of the caustic produced by an evaporating water droplet. Photographs (a)-(f) represent successively later times. After photograph (f), the evolution of the caustic reversed, finally returning to a form resembling photograph (a). (The photographs were taken with different droplets, with slightly different magnifications.)

As shown in Fig. 1, the prominent initial cusp at the bottom of the pattern (a) shrank and retracted (b) to form a fold caustic (c). At the retraction point a feature, which has been called a diffraction star because of its three-pointed starlike shape, ${ }^{10}$ appeared on the fold caustic and rose above it. The diffraction star is not a caustic; rather, it is the interference pattern that sur- rounds the elliptic umbilic caustic when the elliptic umbilic is too small to be readily visible. ${ }^{25}$ As the evolution continued, the diffraction star transformed into a three-cusped elliptic umbilic caustic [Fig. 1(d)]. The elliptic umbilic caustic then grew in size while its upper two cusps dimmed and the lower cusp remained bright and approached the fold caustic [Fig. 1(e)]. 


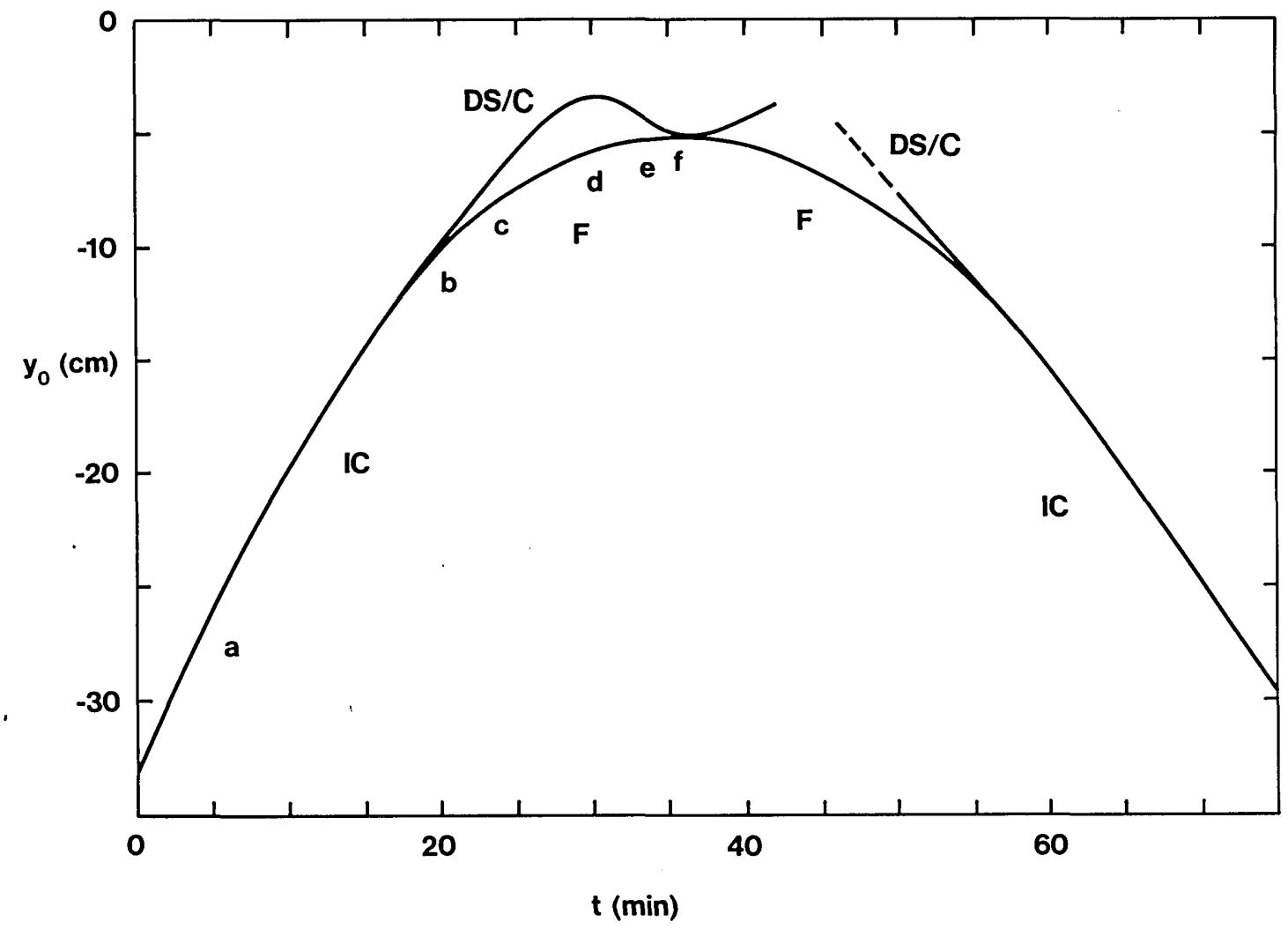

Fig. 2. Location on the viewing screen of the initial cusp point $(I C)$, the bottom of the fold caustic $(F)$, and the diffraction star/elliptic umbilic $(D S / C)$ as a function of time. The points labeled $a-f$ represent the stages of the evolution of the caustic shown in Figs. 1(a)-(f). Shortly after the start of the reversal of the caustic, the cusp inside the fold temporarily disappeared and is thus not shown in the figure. Its return as a dim, featureless patch of light is indicated by the dashed segment. The time at which the dim patch became a recognizable diffraction star corresponds to the end of the dashed segment.

The cusp widened while the fold closed until they merged [Fig. 1(f)]. The entire process then reversed, until the entire caustic pattern once again consisted of several cusps joined by folds as in Fig. 1(a). The full evolution took about an hour. Although Figs. 1(a)-(f) are photographs of the caustics produced by a number of different water droplets and have slightly different magnifications, the caustic evolution was identical for every trial and for each of the three template holes tested. During the reversed phase of the evolution, the surface of the water droplet was outwardly concave, a shape that cannot occur for a raindrop on a window pane or for the geometry considered by Nye, ${ }^{11}$ but which was allowed by the $0.76-\mathrm{mm}$ depth of the template hole.

As the caustic evolved on the screen, the vertical coordinates of the cusp points, the lowest position of the fold caustic, and the center of the diffraction star were measured (Fig. 2). Shortly after the reversal of the caustic began, the cusp grew dim and then vanished for $\sim 5 \mathrm{~min}$. It slowly reappeared as a very dim diffraction star which thereafter increased in brightness and evolved into the final cusp.

As the water droplet slowly evaporated during the caustic evolution, portions of the laser beam were masked between the laser and the water droplet to determine where light rays passed through the droplet surface to produce the different caustic features. The locations of the rays are indicated in Figs. 3(a)-(f). The location responsible for the initial cusp and the diffraction star which grew from its retraction remained at the top of the droplet. The location responsible for the fold that followed the retraction slowly fell from the top to approximately the middle of the droplet. Then the location responsible for the elliptic umbilic and its lowermost cusp similarly but more rapidly fell from the top to the middle. During the reversal of the caustic, the location responsible for the diffraction star quickly dropped from the middle to the bottom of the droplet, but the location responsible for the fold lingered near the middle before it finally slid to the bottom. The location responsible for the final cusp was at the bottom of the droplet.

The focal length of the water droplet as a whole was measured by placing a card at various positions between the droplet and the screen. For Fig. 1(a), the focal length was $\sim 1 \mathrm{~cm}$. By Fig. 1(d) it was $\sim 3 \mathrm{~cm}$, and prior to Fig. 1(e) it was $\sim 5 \mathrm{~cm}$. For Fig. 1(e), the focal length remained at $5 \mathrm{~cm}$ but progressively less of the light passing through the droplet was focused. Just prior to Fig. 1(f) the focusing ceased. During the reversal of the caustic, the droplet had evaporated to such an extent that its surface was concave outward and did not focus the laser light passing through it. 


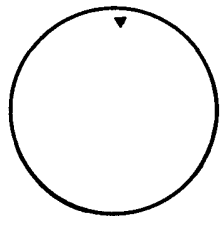

(a)

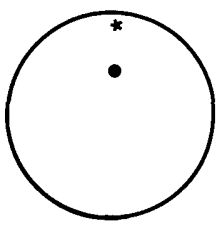

(c)

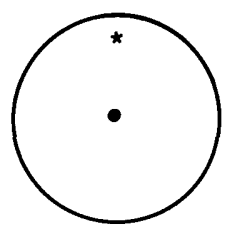

(e)

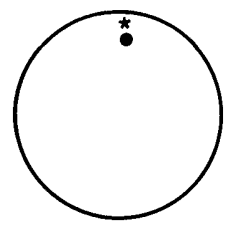

(b)

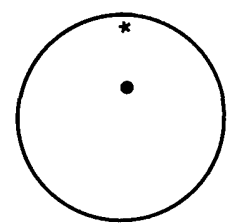

(d)

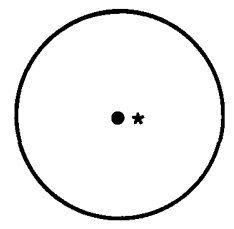

(f)
Fig. 3. Approximate location of the light rays passing through the water droplet which produce the initial cusp point (triangle), the bottom of the fold (circle), and the diffraction star/elliptic umbilic (star) of the caustic in Fig. 1. The droplet cross sections labeled (a)(f) correspond to the stages of the evolution of the caustic shown in Figs. 1(a)-(f).

\section{Analysis of the Refraction Caustic of the Evaporating Water Droplet}

The evolution of the caustic as shown in Figs. 1(a)(f) is typical of a progression through various sections taken through the parabolic umbilic caustic, as organized in Fig. 7 of Ref. 11 and in Fig. 5.24 of Ref. 22 for $\epsilon$ $>0$ and $\eta>0$. As mentioned in Sec. III, the observed caustic is produced by a perturbation in the shape of the surface of the water droplet. We model the droplet surface shape in the following way. The entrance plane $(x, y)$ coordinate system is in the plane of the template with its origin at the center of the template hole. The $y$-axis is vertical and the $x$-axis is horizontal. The center of the surface perturbation is on the $y$ axis at a distance $K$ above the center of the hole. We employ $\left(x_{p}, y_{p}\right)$ as the coordinates of a point on the surface measured with respect to the center of surface perturbation. The two sets of entrance plane coordinates are related by

$$
x=x_{p}, \quad y=y_{p}+K .
$$

We assume that the shape of the droplet as a whole is a portion of a sphere whose radius of curvature is $r_{c}$. The thickness of the water droplet lens is then

$$
t_{c}(x, y)=t_{0}+\left(r_{c}^{2}-x^{2}-y^{2}\right)^{1 / 2}-r_{c} \text {, }
$$

where $t_{0}$ is the thickness of the droplet above the template surface at the center of the hole. The focal length of the water droplet lens is

$$
f_{c}=\frac{r_{c}}{n-1} \text {. }
$$

In the thin lens approximation, $t_{c}(x, y)$ can be expanded in a Taylor series and only the terms quadratic in $x$ and $y$ need be retained. The thin lens approximation is valid for our experiment since at the beginning of the caustic evolution when the curvature of the droplet is the greatest, the focal length is $\sim 1 \mathrm{~cm}$ and $a /$ $r_{c}$ is $<0.5$. As the droplet evaporates, its surface flattens and $a / r_{c}$ decreases further.

Since the caustics in Fig. 1 are indicative of the parabolic umbilic caustic and since the symmetry axis of the caustic in Fig. 1 is vertical, the perturbation in the droplet surface that results from the perimeter irregularity is taken to be of the form

$$
t_{p}\left(x_{p}, y_{p}\right)=\frac{\epsilon}{4(n-1)} y_{p}^{4}+\frac{\eta}{(n-1)} x_{p}^{2} y_{p}-\frac{x_{p}^{2}}{2 r_{x}}-\frac{y_{p}^{2}}{2 r_{y}},
$$

where $\epsilon$ and $\eta$ are constants with the units $\mathrm{m}^{-3}$ and $\mathrm{m}^{-2}$, respectively, and where the $r_{x}$ and $r_{y}$ terms describe an astigmatic perturbation to the underlying spherical cap of Eq. (10) in the immediate neighborhood of the surface irregularity. Equation (12) and the Taylor series expansion of Eq. (10) may be combined to give the total thickness function of the droplet. With this inserted into Eq. (1), the phase function $\phi$ written in terms of $x_{p}$ and $y_{p}$ assumes the form of Eq. (5) for the parabolic umbilic caustic with the identifications

$$
\begin{aligned}
& \alpha=\frac{x_{0}}{z_{0}}, \\
& \beta=\frac{y_{0}}{z_{0}}+\frac{K}{f_{c}}-\frac{K}{z_{0}}, \\
& \gamma=\frac{1}{2}\left[\frac{1}{z_{0}}-\frac{1}{f_{c}}-\frac{(n-1)}{r_{x}}\right], \\
& \delta=\frac{1}{2}\left[\frac{1}{z_{0}}-\frac{1}{f_{c}}-\frac{(n-1)}{r_{y}}\right] .
\end{aligned}
$$

The parameters $\gamma$ and $\delta$ have the units $\mathrm{m}^{-1}$. The parameters $\alpha$ and $\beta$ are dimensionless and are proportional to the $x_{0}$ and $y_{0}$ coordinates on the viewing screen. Thus the appearance of the caustic in $\alpha-\beta$ space is the same as its appearance on the screen, as stated in the preceding section.

The goal of this section is to determine the path of the caustic evolution of Fig. 1 in the $\gamma-\delta$ plane. The first step is to obtain the signs of $K, \epsilon$, and $\eta$ since these signs determine the orientation of the caustic in $\alpha-\beta$ space and the organization of the sections of the caustic at various points in the $\gamma-\delta$ plane. At the beginning of the caustic evolution, the cusp at the bottom of the viewing screen in Fig. 1(a) is produced by light rays passing through the very top of the droplet as shown in Fig. 3(a). Thus the perimeter irregularity that produces the first half of the evolution is located at the top of the droplet and $K$ is initially positive. Since the dominant cusp in Fig. 1(a) points vertically downward, 


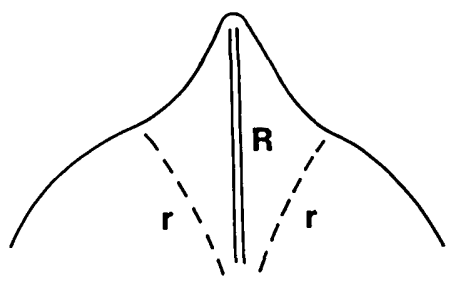

(a)

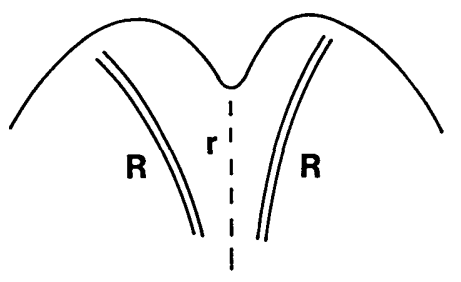

(b)

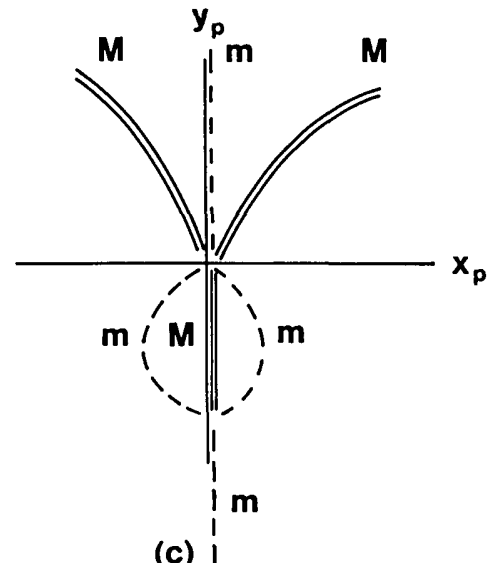

(c) 1

Fig. 4. (a) Finger perimeter irregularity and (b) an indentation perimeter irregularity both shown greatly exaggerated. The double lines $R$ represent surface ridges and the dashed lines $r$ represent surface ravines. The surface perturbation $\epsilon y_{p}^{4} / 4+\eta x_{p}^{2} y_{p}$ with $\epsilon<0$ and $\eta>0$ is shown in (c) as a function of $x_{p}$ and $y_{p}$. The double lines $M$ are relative maxima and the dashed lines $m$ are relative minima.

the graph of Eqs. (7) and (8) shows that the possible signs of the parameters $\epsilon$ and $\eta$ in Eq. (5) are either $\epsilon<$ $0, \eta>0$ or $\epsilon>0, \eta>0$. For the other two choices of signs $\epsilon<0, \eta<0$ and $\epsilon>0, \eta<0$, the graphs of Eqs. (7) and (8) have the cusp pointing upward instead.

After the dominant intitial cusp retracted to form the fold and diffraction star in Fig. 1(c), the location on the droplet responsible for the diffraction star in Fig. 3 (c) was higher than the location responsible for the fold which is consistent with one choice of signs: $\epsilon<0$, $\eta>0$. For this case, the portion of the $H(\phi)=0$ curve on the droplet producing the diffraction star also occurs at a larger $y$ value than does the portion producing the fold. For the other choice of sign, $\epsilon>0$ and $\eta>0$, the fold-producing portion of the $H(\phi)=0$ curve of Eq. (6) occurs higher on the droplet instead.

Physically, the choice of signs for $\epsilon$ and $\eta$ reveals that the irregularity in the droplet perimeter of the droplet which causes the surface shape perturbation is a small indentation rather than a small finger of water extending outward. A finger irregularity at the top of the droplet produces a small ridge on the droplet surface along the $y$-axis, flanked on each side by a small ravine. In contrast, an indentation irregularity at the top of the droplet produces a central ravine that is flanked on each side by a ridge. These two possibilities are shown greatly exaggerated in Figs. 4(a) and (b). The surface perturbation thickness function $\epsilon y_{p}^{4} / 4+\eta x_{p}^{2} y_{p}$ is shown in Fig. 4(c) for $\epsilon\langle 0, \eta\rangle 0$. A comparison between Figs. 4(b) and (c) indicates that the controlling irregularity for the first stage of the caustic evolution is an indentation in the droplet perimeter at the top of the droplet.

The second step in determining the path of the evolution of the caustic in the $\gamma-\delta$ plane is to organize of the sections of the caustic. For our situation with $\epsilon<$ $0, \eta>0$ the point in the $\gamma-\delta$ plane that corresponds to a given caustic section is rotated by $180^{\circ}$ from its position if $\epsilon>0, \eta>0$ as is the case in Fig. 7 of Ref. 11 and in Fig. 5.24 of Ref. 22.

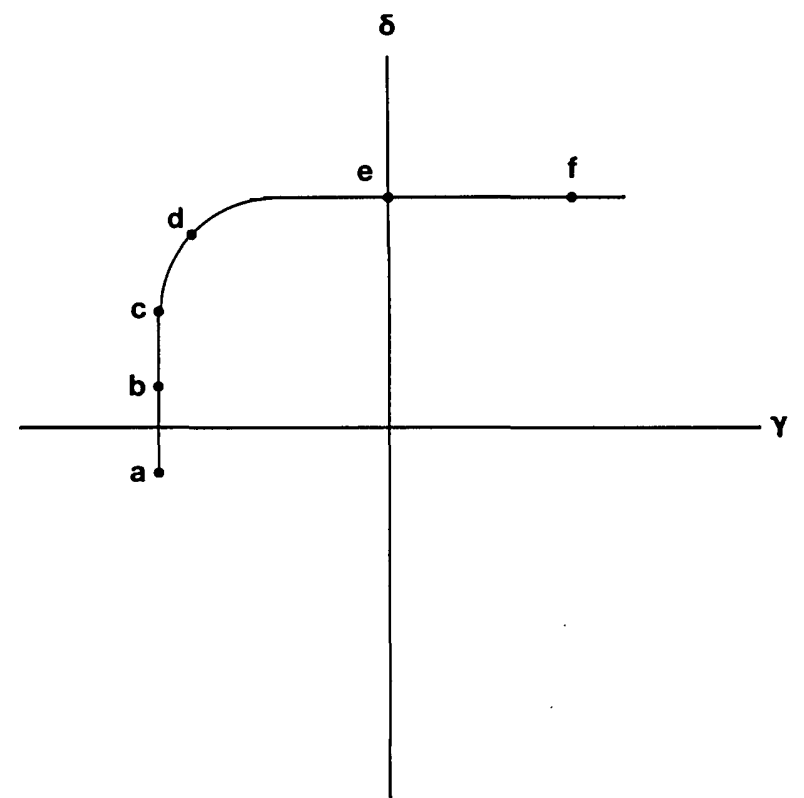

Fig. 5. Path of the first half of the evolution in the $\gamma-\delta$ plane of the caustic produced by the evaporating water droplet. Points $a-f$ correspond to the stages of the evolution of the caustic shown in Figs. 1(a)-(f).

All the data in Figs. 1-3 support the assertion that the path in the $\gamma-\delta$ plane for the first half of the caustic evolution is given by Fig. 5, where the points labeled $a-$ $f$ correspond to the caustics of Figs. 1(a)-(f). Along the initial portion of the path, $\gamma$ is a negative constant while $\delta$ increases from negative to positive. Then after the path changes direction, the value of $\delta$ remains constant while $\gamma$ increases from negative to positive.

To ensure that the caustics in Fig. 1 are consistent with this path, in Figs. 6(a)-(f) we graphed the $H(\phi)=$ 0 curves of Eq. (6) corresponding to the points labeled $a-f$ in Fig. 5. In Figs. 7(a)-(f) we then graphed the 


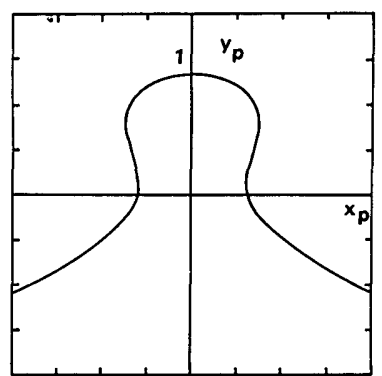

(a)

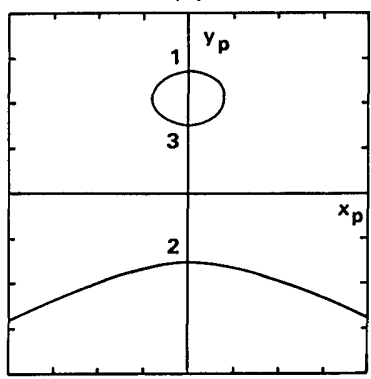

(c)

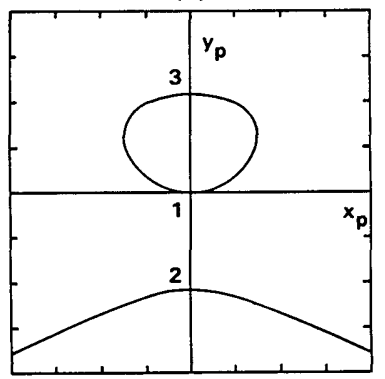

(e)

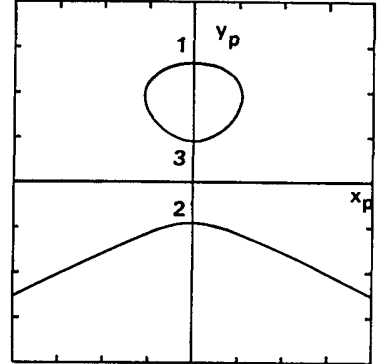

(b)

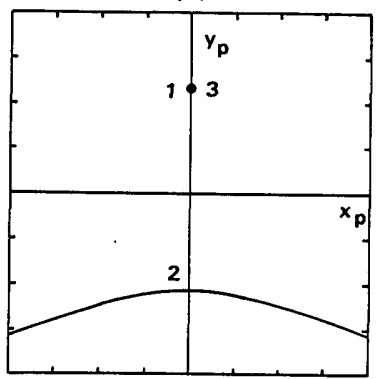

(d)

(f)

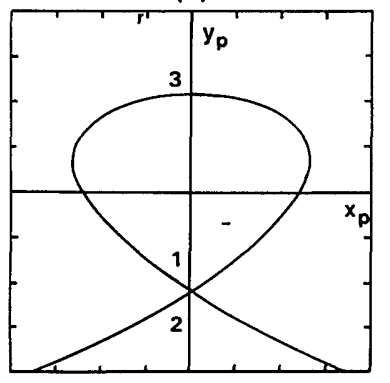

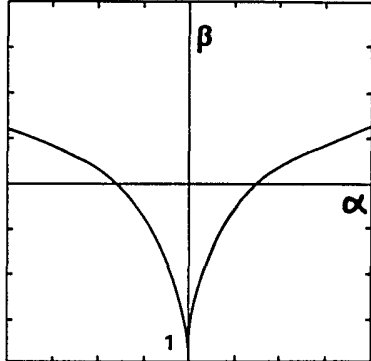

(a)

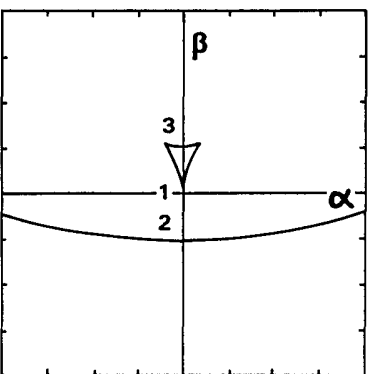

(c)

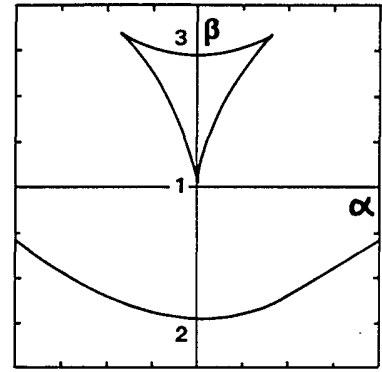

(e)

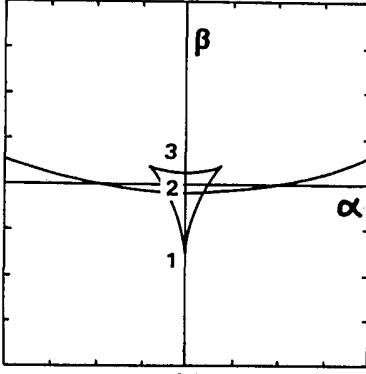

(b)

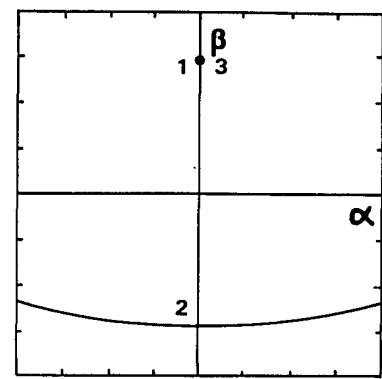

(d)

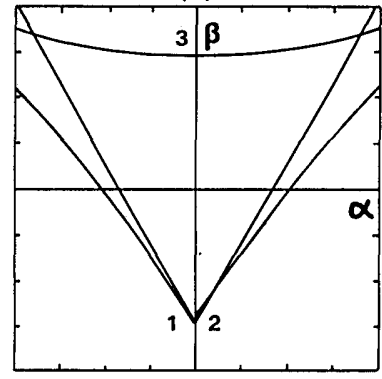

(f)
Fig. 6. The $H(\phi)=0$ curves in the $x_{p}-y_{p}$ plane on the droplet surface for the parabolic umbilic phase function of Eq. (5). The graphs labeled (a) -(f) correspond to the stages of the evolution of the caustic shown in Figs. 1(a)-(f). The points labeled 1-3 are the solutions of Eq. (6) for $x=0$.

caustics derived from the curves via Eqs. (7) and (8). The caustic shapes in Fig. 7 are identical to the appearance of the actual caustics in Fig. 1, except that sometimes an actual caustic is masked by its surrounding interference pattern.

The locations of the fold, cusp points, and diffraction star on the screen given in Fig. 2 also support our choice of the path in the $\gamma-\delta$ plane shown in Fig. 5. In the far field where $z_{0} \gg K$, the vertical coordinate of a given caustic feature on the screen is obtained via Eq. (14) and is approximately given by

$$
y_{0} \approx\left(\beta-\frac{K}{f_{\mathrm{c}}}\right) z_{0} \text {. }
$$

If $K$ remains nearly constant as the water evaporates and as the focal length $f_{c}$ increases, Eq. (17) reveals that an initially negative coordinate $y_{0}$ rises on the screen due to the change in focal length. Before the initial cusp retracts, Figs. 7(a) and (b) show that it is also rising in $\alpha-\beta$ space. Thus, since these changes are in the same direction, the initial cusp rises quickly on the screen (Fig. 2). After the retraction and as the focal length continues to increase, the coordinate $y_{0}$ continues to rise according to Eq. (17). As shown in Figs. $7(\mathrm{c})$ and (d), in $\alpha-\beta$ space the diffraction star/ elliptic umbilic also rises. Again, with the two types of change in the same direction, the caustic moves rapidly upward on the screen. In contrast, the fold drops in $\alpha$ $\beta$ space, and so it moves upward more slowly on the screen. For Figs. 7(e) and (f) when $\gamma$ is variable and $\delta$ is fixed, the surface of the water droplet is nearly flat and the focal length is very large. As a result, the vertical coordinate of a caustic feature on the viewing screen is

$$
y_{0} \approx \beta z_{0} \text {. }
$$

Using Eqs. (6) and (8) with $x=0$ and substituting for $\beta$, 
the vertical coordinate of the fold on the viewing screen is

$$
y_{0} \approx-\frac{4}{3} \delta\left(\frac{2 \delta}{3|\epsilon|}\right)^{1 / 2} z_{0}
$$

which is constant as is observed in Fig. 2 assuming $\epsilon$ remains constant as well.

The vertical coordinate of the interior cusp point on the viewing screen is

$$
y_{0} \approx-\frac{\gamma}{\eta}\left(-|\epsilon| \frac{\gamma^{2}}{\eta^{2}}+2 \delta\right) z_{0} .
$$

This is an increasing function of $\delta$ until the path of the caustic evolution in the $\gamma-\delta$ plane changes direction at point $d$ in Fig. 5. Then the coordinate becomes a decreasing function of $\gamma$ for constant $\epsilon$ and $\eta$ and has a relative minimum when the cusp and the fold merge at

$$
\gamma=\left(\frac{2 \eta^{2} \delta}{3|\epsilon|}\right)^{1 / 2}
$$

(point $f$ in Fig. 2).

The data in Fig. 3 also support the choice of the path for the caustic evolution shown in Fig. 5. The connection between the shape of the caustic and the shape of its $H(\phi)=0$ curve produces the following identifications. The approximate parabolic shape of the $H(\phi)=$ 0 curve near point 1 in Fig. 6(a) corresponds to the cusp at point 1 in Fig. 7(a). The ellipse containing points 1 and 3 in Figs. 6(b)-(e) corresponds to the elliptic umbilic in Figs. 7(b)-(e). The region about point 2 and the bottom of the ellipse in Figs. 6(b)-(e), taken together, resemble a hyperbola and correspond to the presence of both a cusp and fold in Figs. 7(b)-(e), characteristic of the hyperbolic umbilic. Finally, the $x$-shape at points 1 and 2 in Fig. 6(f) corresponds to the singular section of the hyperbolic umbilic at points 1 and 2 in Fig. 7(f). The migration of the locations on the $H(\phi)=$ 0 curve in Fig. 6 that correspond to the initial cusp, the fold, and the diffraction star/elliptic umbilic is identical to the migration of the locations on the droplet that are summarized in Fig. 3.

After Fig. 1(f), the caustic on the screen reversed its progression until it again resembled Fig. 1(a). At Fig. 1 (f) or shortly after, control of the caustic was exchanged from the indentation perimeter irregularity at the top of the droplet to another perimeter irregularity at the bottom of the droplet. Thus $K$ quickly decreased from positive to negative at about the same time that the general droplet outward curvature changed from convex to concave. From the exchange point onward in the evolution, with $K$ and $f_{c}$ being both negative, the dominant caustic features located according to Eq. (17) still occurred at the bottom of the screen. An analysis identical to that employed for the first half of the evolution shows that after the exchange of control and during the caustic reversal (i) the signs of $\epsilon$ and $\eta$ are both positive; (ii) the dominant perimeter irregularity at the bottom of the droplet is a finger of water that extends downward; (iii) the organization of the caustic in the $\gamma-\delta$ plane is the case treated in Refs. 11 and 22; and (iv) the path of the last half of the

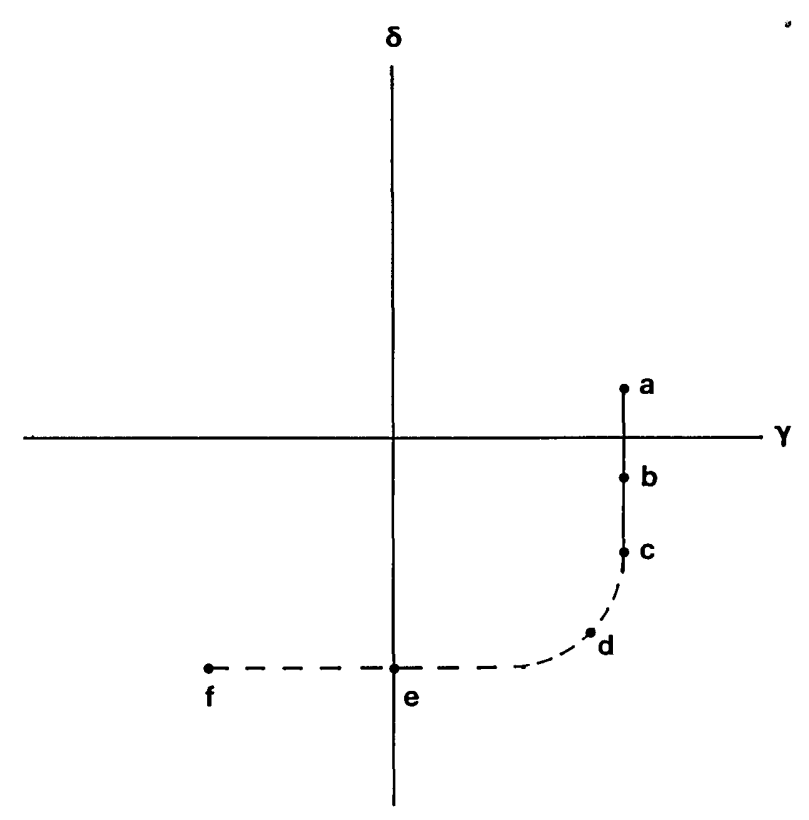

Fig. 8. Path of the second half of the evolution in the $\gamma-\delta$ plane of the caustic produced by the evaporating water droplet. The dashed portion of the path can only be inferred since it corresponds to the time interval during which the cusp caustic temporarily disappeared as indicated in Fig. 2.

evolution of the caustic in this new $\gamma-\delta$ plane is given in Fig. 8. The portion of the path corresponding to the temporary vanishing of the interior cusp is indicated by the dashed segment.

\section{v. Changes in the Surface Curvature of an Evaporating Water Droplet}

In this section we relate the caustic parameters $\gamma$ and $\delta$ of Eqs. (15) and (16) to the perturbation in the shape of the droplet surface that is produced by the controlling perimeter irregularity. Since the caustics are in the far field with $z_{0} \approx 2 m$ and with $f_{c}$ initially being about a centimeter, the $1 / z_{0}$ terms in Eqs. (15) and (16) can be neglected. Such neglect is not justified for the portion of the evolution near Fig. 1(f) where (i) the focal length became comparable to $z_{0}$, (ii) the control of the caustic changed from an upper perimeter irregularity to one at the bottom, and (iii) when the evolutionary path switched from Fig. 5 to Fig. 8 . Near the center of the perturbation the droplet curvature in the horizontal $x-z$ plane is the sum of the $f_{c}$ and $r_{x}$ contributions. In the vertical $y-z$ plane it is the sum of the $f_{c}$ and $r_{y}$ contributions. Thus from Eqs. (15) and (16) sign changes of $\gamma$ and $\delta$ correspond to sign changes in the curvature of the droplet.

The caustic path in Fig. 5 suggests that, as the droplet evaporates, the surface curvature near the top of the droplet does not change isotropically. Initially $\gamma$ and $\delta$ are both negative and thus the top of the droplet is convex outward in both the $x-z$ and $y-z$ planes. When evaporation first begins, while $\gamma$ is constant and $\delta$ is varying, the curvature in the horizontal $x-z$ plane remains convex outward and the curvature in the vertical $y-z$ plane changes from convex outward, to flat, to 
concave outward. Then, while $\delta$ is constant and $\gamma$ is varying, the curvature in the $y-z$ plane remains concave outward and the curvature in the $x-z$ plane changes from convex outward, to flat, to concave outward. This progression of surface shape of the upper half of the droplet is from a relative maximum $(\gamma<0, \delta$ $<0)$, to a saddle point $(\gamma<0, \delta>0)$, to a relative minimum $(\gamma>0, \delta>0)$. During the first part of the evolution, the slight gravitational sagging of the droplet keeps the curvature of the lower portion of the droplet convex outward in both the $x-z$ and $y-z$ planes.

With the top and bottom of the droplet having opposite curvatures at this stage of the evolution, the droplet as a whole is nearly flat on the average, and it ceases to focus light effectively. This is the time at which the control of the caustic transfers from the perimeter irregularity at the top of the droplet to one at the bottom. Upon further evaporation, more of the surface of the droplet sinks into the template hole. With the top of the droplet already being concave outward, the variations of $\gamma$ and $\delta$ in Fig. 8 suggest that near the bottom of the droplet the curvature in the vertical $y-z$ plane remains convex outward while the curvature in the horizontal $x-z$ plane changes from convex outward, to flat, to concave outward. This change on the lower portion of the droplet may, in fact, have begun while the curvature of the $x-z$ plane was changing on the top half of the droplet. Lastly, the curvature in the $y-z$ plane near the bottom of the droplet changes from convex outward, to flat, to concave outward. The entire droplet is finally concave outward at the end of the evaporation sequence.

\section{Summary}

Evaporation of liquid drop lenses provides a good test case in which caustics may be employed to provide surface shape information. Since a number of different surfaces can produce similar-looking caustics, measurements of other quantities, such as the locations of the caustic features on the viewing screen and the locations on the surface responsible for the caustic features, provide valuable information for unambiguously determining the surface shape.

\section{References}

1. M. Herzberger, "Light Distribution in an Optical Image," J. Opt. Soc. Am. 37, 485-493 (1947).

2. M. Herzberger, Modern Geometrical Optics (Wiley-Interscience, New York, 1958), p. 156.

3. O. N. Stavroudis, The Optics of Rays, Wavefronts, and Caustics (Academic, New York, 1972), p. 159.
4. J. B. Keller and H. B. Keller, "Determination of Reflected and Transmitted Fields by Geometrical Optics," J. Opt. Soc. Am. 40, 48-52 (1950).

5. D. G. Burkhard and D. L. Shealy, "Flux Density for Ray Propagation in Geometrical Optics," J. Opt. Soc. Am. 63, 299-304 (1973).

6. O. N. Stavroudis and R. C. Fronczak, "Caustic Surfaces and the Structure of the Geometrical Image," J. Opt. Soc. Am. 66, 795800 (1976).

7. D. G. Burkhard and D. L. Shealy, "Simplified Formula for the Illuminance in an Optical System," Appl. Opt. 20, 897-909 (1981).

8. M. V. Berry, "Waves and Thom's Theorem," Adv. Phys. 25, 1-26 (1976).

9. M. V. Berry and C. Upstill, "Catastrophe Optics: Morphologies of Caustics and Their Diffraction Patterns," Prog. Opt. 18, 257 346 (1980).

10. J. F. Nye, "Optical Caustics in the Near Field from Liquid Drops," Proc. R. Soc. London Ser. A 361, 21-41 (1978).

11. J. F. Nye, "Optical Caustics from Liquid Drops Under Gravity: Observations of the Parabolic and Symbolic Umbilics," Philos. Trans. R. Soc. London Ser. A 292, 4-44 (1979).

12. P. L. Marston and E. H. Trinh, "Hyperbolic Umbilic Diffraction Catastrophe and Rainbow Scattering from Spheroidal Drops," Nature London 312, 529-531 (1984).

13. J. F. Nye, "Rainbow Scattering from Spheroidal Drops-an Explanation of the Hyperbolic Umbilic Foci," Nature London 312, 531-532 (1984).

14. J. F. Nye, "The Catastrophe Optics of Liquid Drop Lenses," Proc. R. Soc. London Ser. A 403, 1-26 (1986).

15. M. V. Berry, "Disruption of Images: the Caustic Touching Theorem," J. Opt. Soc. Am. A 4, 561-569 (1987).

16. G. Dangelmayr and F. J. Wright, "On the Validity of the Paraxial Eikonal in Catastrophe Optics," J. Phys. A Gen. Phys. 17, 99-108 (1984).

17. E. Bochove, "Geometrical Optics Field of General Optical Systems," J. Opt. Soc. Am. 69, 891-897 (1979).

18. P. S. Theocaris and J. G. Michopoulos, "Generalization of the Theory of Far-Field Caustics by the Catastrophe Theory," Appl. Opt. 21, 1080-1091 (1982).

19. J. D. Walker, "Caustics: Mathematical Curves Generated by Light Shined Through Rippled Plastic," Sci. Am. 249(3), 190201 (1983).

20. J. A. Lock and J. H. Andrews, "Caustics Associated with Cubic Phase Functions," J. Opt. Soc. Am., submitted.

21. J. A. Lock and J. D. Walker, "In Search of the Transverse Swallowtail Caustic," J. Opt. Soc. Am., submitted.

22. R. Thom, Structural Stability and Morphogenesis (Benjamin, Reading, MA, 1975), pp. 81-90 and 189-191.

23. J. D. Walker, "A Drop of Water Becomes a Gateway into the World of Catastrophe Optics," Sci. Am. 261(3), 176-179 (1989).

24. M. Minnaert, The Nature of Light and Color in the Open Air (Dover, New York, 1954), pp. 167-169.

25. M. V. Berry, J. F. Nye, and F. J. Wright, "The Elliptic Umbilic Diffraction Catastrophe," Philos. Trans. R. Soc. London 291, 453-484 (1979). 